\title{
Prevalência de obesidade associada à ingestão calórica, glicemia e perfil lipídico em uma amostra populacional de idosos do Sul do Brasil
}

\author{
Obesity prevalence associated with caloric intake, glycemia, and serum lipides in a sample \\ population of elderly adults from Southern Brazil
}

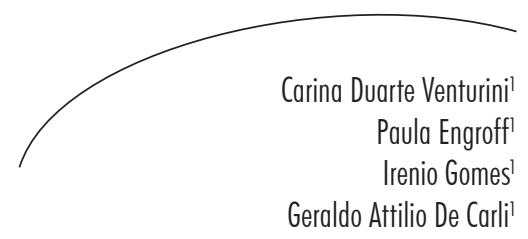

\section{Resumo}

Objetivo: Determinar a prevalência de obesidade associada ao consumo de macronutrientes, às alterações do perfil lipídico, à glicemia e à prática de atividade física em idosos. Métodos: Foi realizado estudo transversal com 304 idosos do município de Porto Alegre, RS, Brasil. Medidas antropométricas de peso e altura foram utilizadas para o cálculo do índice de massa corporal (IMC) e foram analisados o perfil lipídico, a glicemia, a ingestão diária de macronutrientes e a prática de atividade física. Resultados: A prevalência de obesidade foi de 30,6\%, sofrendo redução com o aumento da idade. As idosas obesas apresentaram maior frequência de hipertrigliceridemia, cujos valores aumentaram conforme o aumento do IMC. Nesse grupo, a prática de atividade física foi menor. Entre os homens, houve maior consumo de proteína na dieta. Conclusão: Os resultados mostraram que a obesidade é um importante problema de saúde na Região Sul do Brasil, sofrendo influência de fatores socioculturais e econômicos que prejudicam a manutenção de uma alimentação saudável. Políticas públicas devem ser direcionadas a fim de controlar esse problema, já que a obesidade é um fator limitante para a longevidade.

\section{Abstract}

Objective: To determine the prevalence of obesity in elderly adults associated with macronutrient consumption, lipid profile, blood glucose and physical activity. Methods: A cross-sectional-study was made with 304 elderly patients from Porto Alegre, State of Rio Grande do Sul, Brazil. Anthropometric measures of weight and height were used to calculate body mass index (BMI), and lipid profile, blood glucose, daily intake of nutrients, and physical activity were analyzed. Results: The prevalence of obesity was 30.6\%, decreasing with increased age. Obese women had higher frequency of hypertriglyceridemia

Palavras-chave: Obesidade. Idoso. Ingestão de Energia. Testes Hematológicos.
Key words: Obesity. Aged. Energy Intake. Hematologic Tests.

\footnotetext{
Instituto de Geriatria e Gerontologia. Pontifícia Universidade Católica do Rio Grande do Sul. Porto Alegre, RS, Brasil.
} 
and these values increased with increasing BMI. In this group, physical activity was lower. Among men, there was higher intake of dietary protein. Conclusion: The results show that obesity is an important health problem in Southern Brazil, being influenced by socio-cultural and economic factors that make it difficult to keep a healthy diet. Public policies should be adopted to control that problem, since obesity is a limiting factor for longevity.

\section{INTRODUÇÃO}

Com o fenômeno de envelhecimento populacional, verificam-se mudanças no perfil epidemiológico, aumentando a necessidade de conhecimento dos fatores que incidem sobre a prevalência das doenças crônico-degenerativas associadas à idade. ${ }^{1}$ Estudos epidemiológicos realizados no Brasil e no mundo apontam importantes transformações demográficas e socioeconômicas nos padrões de atividade física e no comportamento alimentar, ocorridas nas últimas décadas. ${ }^{2}$ Há relatos de que a automação, a tecnologia e o estilo de vida mais confortável desencadeiam comportamentos sedentários. ${ }^{3}$ Nesse contexto, mudanças nos indicadores nutricionais da população brasileira também foram observadas, especialmente no que se refere ao incremento da obesidade. ${ }^{4}$

A obesidade é uma doença crônica, multifatorial, definida como excesso de gordura corporal. Sua etiologia pode estar relacionada a ingestão alimentar excessiva e pouco saudável, sedentarismo, fatores genéticos, metabólicos, socioculturais e psicossociais. $^{5}$ As medidas antropométricas representadas pelo índice de massa corporal (IMC), razão cintura-quadril (RCQ) e circunferência abdominal (CA) representam uma maneira racional e eficiente de se presumir o volume e a distribuição de gordura. ${ }^{6}$ É considerado obeso o indivíduo que apresenta IMC maior ou igual a $30 \mathrm{~kg} / \mathrm{m}^{2}{ }^{5}$ A definição de obesidade não se diferencia na população idosa, apesar da tolerância maior dos idosos com o aumento de IMC, podendo assim a obesidade ser definida em um patamar de IMC mais elevado nessa faixa etária.

Estudos epidemiológicos sobre o estado nutricional em idosos indicam que os distúrbios nutricionais estão associados a um maior risco de morbidade e mortalidade. ${ }^{8}$ A prevalência de pessoas com sobrepeso e obesidade também está aumentando na população. No Brasil, a prevalência de obesidade é de 36,9\%, conforme dados da Organização Mundial da Saúde (OMS). ${ }^{?}$ Segundo o Instituto Brasileiro de Geografia e Estatística (IBGE), a prevalência de obesidade no Rio Grande do Sul é de 29,8\%, e em Porto Alegre, $21,7 \%{ }^{10}$

O aumento da prevalência de sobrepeso em adultos pode se refletir na população idosa, pois, com o envelhecimento, ocorrem alterações na composição corporal, tais como redistribuição da gordura corporal, diminuição da massa muscular e óssea. É evidente o incremento de tecido adiposo em detrimento da massa muscular, sendo esse aumento mais pronunciado no abdome (obesidade abdominal), especialmente em vísceras. ${ }^{11}$

$\mathrm{Na}$ velhice, a obesidade está associada ao aumento da mortalidade e dos riscos de diabetes mellitus tipo 2, intolerância à glicose, aterosclerose, hipertensão arterial, dislipidemia, acidente vascular cerebral, coronariopatias, colelitíase, osteoartrose, alguns tipos de câncer, declínio funcional e invalidez. Idosos obesos têm pior qualidade de vida que idosos não obesos, o que é atribuído ao estilo de vida não saudável e às doenças crônicas causadas pela obesidade..$^{11-13}$

Tendo em vista a alta prevalência de sobrepeso e obesidade na população e os problemas de saúde associados, este estudo se propôs a determinar a prevalência de obesidade por sexo em idosos de Porto Alegre e sua associação com o consumo de macronutrientes, as alterações dos exames bioquímicos e a prática de atividade física. 


\section{MÉTODOS}

Foi realizado estudo transversal, de base populacional, pelo Instituto de Geriatria e Gerontologia (IGG) da Pontifícia Universidade Católica do Rio Grande do Sul (PUCRS), em parceria com a Prefeitura Municipal de Porto Alegre, no período de dezembro de 2005 a julho de 2006 (Estudo Multidimensional dos Idosos de Porto Alegre - EMIPOA). Foi estudada uma amostra aleatória de indivíduos residentes no município de Porto Alegre, com idade igual ou superior a 60 anos, em duas etapas: entrevista domiciliar e avaliação multidisciplinar.

Para o cálculo do tamanho amostral, realizado no projeto original para estimativa de prevalências, foi considerada uma margem aceitável para um intervalo de confiança de 95\% de: (1) 1\% para prevalências abaixo de 1\% e acima de $99 \%$; (2) 2\% para prevalências entre 1 e $5 \%$ e entre 95 e 99\%; (3) 3\% para prevalências entre 5 e $10 \%$ e entre 90 e $95 \%$; e (4) $5 \%$ para prevalências entre 20 e $80 \%$. Utilizando-se o programa Sample.exe do pacote estatístico PEPI versão 4, obteve-se como maior valor de tamanho amostral 447 idosos. A seleção foi realizada de forma aleatória por residência, estratificada pelas regiões censitárias do IBGE. Foram avaliados 512 idosos de forma multidisciplinar, do grupo inicial. Foram realizadas 1.078 entrevistas domiciliares, prevendo uma participação posterior de metade dos entrevistados.

Os idosos foram avaliados por profissionais de diferentes áreas, incluindo Medicina, Educação Física, Enfermagem e Nutrição. Também foi realizada coleta de sangue para subsequente análise da glicemia e do perfil lipídico dos idosos, cujas dosagens bioquímicas foram realizadas no Laboratório de Patologia Clínica do Hospital São Lucas da PUCRS. Como critério de inclusão, foram utilizados os indivíduos idosos cujas informações sobre dados antropométricos, nutricionais, laboratoriais e de atividade física estivessem completas no banco de dados.

No presente estudo, da amostra de 512 idosos, foi realizada uma análise de 304 indivíduos, sendo 87 homens e 217 mulheres. O IMC foi estimado dividindo-se o peso $(\mathrm{em} \mathrm{Kg}$ ) pelo quadrado da altura (em m) e foram considerados os seguintes pontos de corte: normal, IMC entre 18,5 e $24,9 \mathrm{Kg} / \mathrm{m}^{2}$; sobrepeso, IMC entre 25,0 e $29,9 \mathrm{Kg} / \mathrm{m}^{2}$; obeso, IMC $\geq 30,0 \mathrm{Kg} / \mathrm{m}^{2}{ }^{5}$ Para análise da glicemia de jejum, consideraram-se como hiperglicemia valores séricos de glicose $>110 \mathrm{mg} / \mathrm{dL}$. Para análise do perfil lipídico, foram considerados alterados os seguintes valores: hipertrigliceridemia para triglicerídeos $\geq 150 \mathrm{mg} / \mathrm{dL}$; hipercolesterolemia para colesterol total $>200 \mathrm{mg} / \mathrm{dL}$; LDL alto para valores de LDL $\geq 160 \mathrm{mg} / \mathrm{dL}$; HDL baixo para valores de HDL $<40$ para homens e $<50$ para mulheres.

A análise da ingestão diária de macronutrientes (carboidrato, proteína e lipídeo), foi realizada por meio do inquérito recordatório de 24 horas, que define a quantidade de alimentos ingeridos nas 24 horas do dia anterior à entrevista, e da investigação da história dietética, que avalia o consumo alimentar nos últimos três meses, mostrando o hábito alimentar do indivíduo.

Todas as análises foram realizadas utilizando o programa estatístico SPSS, versão 17. Os resultados foram expressos em frequência, média e desvio-padrão, sendo considerado significativo $\mathrm{o}$ valor de $\mathrm{p}<0,05$. A fim de verificar a associação entre as variáveis estudadas e o estado nutricional, foram utilizados os testes estatísticos: quiquadrado, para comparar a frequência dos exames bioquímicos alterados e a prática de atividade física com o estado nutricional; análise de variância (ANOVA), para comparar as médias dos exames bioquímicos e o consumo alimentar entre os grupos de indivíduos classificados de acordo com o estado nutricional. O teste Post Hoc de Bonferroni foi empregado para verificar a significância entre dois grupos.

$\mathrm{Na}$ análise multivariada, foi utilizada regressão logística múltipla, sendo o critério de entrada todas as variáveis, exceto colesterol total, pois está diretamente relacionado com HDL e LDL. No consumo dos macronutrientes, foi criada a variável proteína $>20 \%$, pois foi a única com resultado significativo na análise bivariada. Para 
o resultado final, as variáveis menos associadas foram retiradas uma a uma, sendo elas o HDL e o consumo de proteína $>20 \%$. Foram mantidas no resultado final as variáveis com $\mathrm{p}<0,20$.

Este estudo foi aprovado pelo Comitê de Ética em Pesquisa da PUCRS sob parecer no 0502935. Todos os participantes assinaram o Termo de Consentimento Livre e Esclarecido.

\section{RESULTADOS}

Dos 304 idosos existentes no banco de dados analisado, 217 (71,4\%) eram mulheres e 87
$(28,6 \%)$, homens. A distribuição entre as faixas etárias revelou maior proporção de indivíduos entre 60 e 69 anos (48,4\%), reduzida com o avançar da idade: $38,2 \%$ tinham entre 70 e 79 anos e $13,5 \%$ possuíam 80 anos ou mais.

De acordo com a classificação do IMC, somente 28\% dos idosos de Porto Alegre apresentaram estado nutricional normal (tabela 1). A prevalência de obesidade nessa população foi de 30,6\%, sendo maior nas mulheres. A análise do estado nutricional por faixa etária revelou que a prevalência de obesidade foi maior na faixa etária de 60 a 69 anos, diminuindo nas faixas etárias mais avançadas $(\mathrm{p}=0,023)$ (tabela 1).

Tabela 1 - Distribuição do estado nutricional de acordo com o sexo e a faixa etária em 304 idosos do município de Porto Alegre no ano de 2006. Porto Alegre-RS, 2006.

\begin{tabular}{lcccc}
\hline Variável & $\begin{array}{c}\text { Normal } \\
\mathrm{n}(\%)\end{array}$ & $\begin{array}{c}\text { Sobrepeso } \\
\mathrm{n}(\%)\end{array}$ & $\begin{array}{c}\text { Obeso } \\
\mathrm{n}(\%)\end{array}$ & $\mathrm{p}^{*}$ \\
\hline $\begin{array}{l}\text { Sexo } \\
\text { Homens }\end{array}$ & $28(32,2)$ & $41(47,1)$ & $18(20,7)$ & \\
$\quad$ Mulheres & $57(26,3)$ & $85(39,2)$ & $75(34,6)$ & 0,060 \\
Faixa etária (anos) & & & & \\
$\quad 60-69$ & $32(21,8)$ & $61(41,5)$ & $54(36,7)$ & 0,023 \\
$70-79$ & $35(30,2)$ & $48(41,4)$ & $33(28,4)$ & \\
$\geq 80$ & $18(43,9)$ & $17(41,5)$ & $6(14,6)$ & \\
Total & $85(28,0)$ & $126(41,4)$ & $93(30,6)$ & \\
\hline
\end{tabular}

*Valores de p baseados no teste qui-quadrado.

A tabela 2 mostra as médias dos exames de glicemia e do perfil lipídico, e a média de consumo de macronutrientes de acordo com a classificação do IMC nos idosos do sexo masculino e feminino. A média da glicemia de jejum não apresentou diferença estatisticamente significativa entre as três categorias de IMC. Os resultados do perfil lipídico mostraram que as mulheres obesas apresentaram em média valores de triglicerídeos maiores que as mulheres com IMC normal (Bonferroni: $\mathrm{p}=0,041$ ). A análise do consumo diário de macronutrientes mostrou que homens obesos consumiam, em média, maior quantidade de proteína na dieta, quando comparados aos idosos com IMC normal (Bonferroni: $\mathrm{p}=0,031$ ). 
Tabela 2 - Médias dos resultados de exames bioquímicos e de consumo de macronutrientes por idosos, classificados segundo o índice de massa corporal em 304 idosos do município de Porto Alegre/RS no ano de 2006. Porto Alegre-RS, 2006.

\begin{tabular}{|c|c|c|c|c|c|c|c|}
\hline \multirow{2}{*}{ Variável } & \multicolumn{2}{|r|}{ Normal } & \multicolumn{2}{|c|}{ Sobrepeso } & \multicolumn{2}{|r|}{ Obeso } & \multirow{2}{*}{$\mathrm{p}^{*}$} \\
\hline & $\mathrm{n}$ & $(\mathrm{m} \pm \mathrm{dp})$ & $\mathrm{n}$ & $(\mathrm{m} \pm \mathrm{dp})$ & $\mathrm{n}$ & $(\mathrm{m} \pm \mathrm{dp})$ & \\
\hline \multicolumn{8}{|l|}{ HOMENS } \\
\hline Glicemia (mg/L) & 27 & $113,2 \pm 53,8$ & 41 & $106,5 \pm 36,9$ & 17 & $105,1 \pm 26,7$ & 0,758 \\
\hline Colesterol total (mg/dL) & 26 & $180,0 \pm 26,9$ & 37 & $184,7 \pm 33,6$ & 18 & $197,2 \pm 43,8$ & 0,255 \\
\hline HDL (mg/L) & 26 & $40,3 \pm 8,5$ & 37 & $41,8 \pm 10,8$ & 18 & $40,9 \pm 10,8$ & 0,846 \\
\hline $\mathrm{LDL}(\mathrm{mg} / \mathrm{L})$ & 26 & $109,1 \pm 24,3$ & 37 & $111,9 \pm 29,3$ & 18 & $118,5 \pm 44,4$ & 0,624 \\
\hline Triglicerídeos (mg/L) & 26 & $153,6 \pm 77,1$ & 37 & $155,7 \pm 69,8$ & 18 & $190,4 \pm 149,3$ & 0,377 \\
\hline \multicolumn{8}{|l|}{ Macronutrientes } \\
\hline Carboidratos (\%) & 27 & $57,5 \pm 11,9$ & 40 & $54,9 \pm 11,0$ & 18 & $50,4 \pm 19,2$ & 0,219 \\
\hline Proteínas (\%) & 27 & $18,4 \pm 5,1$ & 40 & $20,4 \pm 6,0$ & 18 & $24,4 \pm 12,1$ & 0,036 \\
\hline Lipídeos (\%) & 27 & $24,1 \pm 11,7$ & 40 & $24,7 \pm 9,8$ & 18 & $25,3 \pm 14,2$ & 0,940 \\
\hline \multicolumn{8}{|l|}{ MULHERES } \\
\hline Glicemia (mg/L) & 56 & $109,0 \pm 47,7$ & 79 & $103,5 \pm 29,5$ & 70 & $121,2 \pm 68,0$ & 0,099 \\
\hline Colesterol total $(\mathrm{mg} / \mathrm{dL})$ & 54 & $217,7 \pm 36,4$ & 76 & $214,1 \pm 39,9$ & 67 & $227,4 \pm 43,1$ & 0,130 \\
\hline HDL (mg/L) & 54 & $50,1 \pm 13,1$ & 76 & $47,1 \pm 12,0$ & 66 & $46,0 \pm 10,1$ & 0,156 \\
\hline LDL (mg/L) & 54 & $137,6 \pm 31,6$ & 75 & $131,7 \pm 36,7$ & 66 & $142,5 \pm 36,9$ & 0,196 \\
\hline Triglicerídeos (mg/L) & 54 & $149,7 \pm 73,9$ & 76 & $177,4 \pm 101,4$ & 64 & $194,5 \pm 109,1$ & 0,046 \\
\hline \multicolumn{8}{|l|}{ Macronutrientes } \\
\hline Carboidratos (\%) & 55 & $58,1 \pm 10,6$ & 78 & $56,0 \pm 12,4$ & 71 & $55,7 \pm 11,2$ & 0,480 \\
\hline Proteínas (\%) & 55 & $17,4 \pm 5,4$ & 78 & $18,7 \pm 6,7$ & 71 & $18,7 \pm 6,1$ & 0,408 \\
\hline Lipídeos (\%) & 55 & $24,7 \pm 9,8$ & 78 & $25,2 \pm 9,3$ & 71 & $25,6 \pm 9,7$ & 0,859 \\
\hline
\end{tabular}

*Valores de $\mathrm{p}$ baseados no teste ANOVA; (m \pm dp): média \pm desvio padrão. 
Em relação às principais alterações laboratoriais encontradas nos idosos com obesidade, foi observado que, nos homens, $56 \%$ apresentaram HDL baixo e 50\%, hipertrigliceridemia. Entre as mulheres obesas, $70 \%$ apresentaram hipercolesterolemia; $67 \%$, HDL abaixo do esperado; e 64\%, hipertrigliceridemia (tabela 3). A hipertrigliceridemia foi mais prevalente entre as mulheres obesas, quando comparadas com as que apresentaram IMC normal ou sobrepeso $(\mathrm{p}=0,019)$. Nos demais exames bioquímicos avaliados, não houve diferença significativa entre os grupos de idosos com IMC normal, sobrepeso e obeso.

Em relação à prática de atividade física, não houve diferença entre os idosos com IMC normal, sobrepeso e obeso. Entretanto, as mulheres praticam menos atividade física do que os homens e, entre estes, observou-se a tendência em praticar menos atividade física nos obesos $(\mathrm{p}=0,085)$.

Tabela 3 - Frequência de exames bioquímicos alterados e sedentarismo, classificados segundo o índice de massa corporal em 304 idosos do município de Porto Alegre no ano de 2006. Porto Alegre-RS, 2006.

\begin{tabular}{lccccc}
\hline \multicolumn{1}{c}{ Variável } & $\mathrm{n}$ & $\begin{array}{c}\text { Normal } \\
\mathrm{n}(\%)\end{array}$ & $\begin{array}{c}\text { Sobrepeso } \\
\mathrm{n}(\%)\end{array}$ & $\begin{array}{c}\text { Obeso } \\
\mathrm{n}(\%)\end{array}$ & $\mathrm{p}^{*}$ \\
\hline HOMENS & & & & & \\
$\quad$ Hiperglicemia & 85 & $5(18,5)$ & $11(26,8)$ & $5(29,4)$ & 0,651 \\
Hipercolesterolemia & 81 & $6(23,1)$ & $10(27,0)$ & $7(38,9)$ & 0,504 \\
HDL reduzido & $14(53,8)$ & $20(54,1)$ & $10(55,6)$ & 0,993 \\
LDL elevado & 11 & $1(3,8)$ & $3(8,1)$ & $3(16,7)$ & 0,326 \\
Hipertrigliceridemia & 81 & $11(42,3)$ & $18(48,6)$ & $9(50,0)$ & 0,846 \\
Sedentarismo & 87 & $11(39,3)$ & $15(36,6)$ & $12(66,7)$ & 0,085 \\
MULHERES & & & & & \\
Hiperglicemia & 205 & $12(21,4)$ & $18(22,8)$ & $23(32,9)$ & 0,253 \\
Hipercolesterolemia & 197 & $40(74,1)$ & $48(63,2)$ & $47(70,1)$ & 0,393 \\
HDL reduzido & 196 & $28(51,9)$ & $48(63,2)$ & $44(66,7)$ & 0,230 \\
LDL elevado & 195 & $10(18,5)$ & $19(25,3)$ & $23(34,8)$ & 0,125 \\
Hipertrigliceridemia & 194 & $21(38,9)$ & $36(47,4)$ & $41(64,1)$ & 0,019 \\
Sedentarismo & 217 & $37(64,9)$ & $55(64,7)$ & $49(65,3)$ & 0,996 \\
\hline
\end{tabular}

*Valores de p baseados no teste qui-quadrado.

A Tabela 4 descreve as variáveis que se associaram de forma independente com obesidade: faixa etária e hipertrigliceridemia.
Houve tendência na associação com sobrepeso para a faixa etária e com obesidade para sedentarismo e LDL alto. 
Tabela 4 - Resultado final das regressões logísticas múltiplas, considerando-se dois diferentes pontos de corte para a variável dependente (classificação dicotômica do IMC) em 304 idosos do município de Porto Alegre no ano de 2006. Porto Alegre-RS, 2006.

\begin{tabular}{|c|c|c|c|c|c|c|}
\hline \multirow{2}{*}{ Variável } & \multicolumn{2}{|c|}{ Sobrepeso } & \multirow[b]{2}{*}{$\mathrm{p}^{*}$} & \multicolumn{2}{|c|}{ Obesidade } & \multirow[b]{2}{*}{$\mathrm{p}^{*}$} \\
\hline & OR & IC $95 \%$ & & OR & IC 95\% & \\
\hline \multicolumn{7}{|l|}{ Sexo } \\
\hline Feminino & 1 & --- & & 1 & --- & \\
\hline Masculino & 1,20 & $0,62-2,30$ & 0,588 & 0,80 & $0,37-1,76$ & 0,586 \\
\hline \multicolumn{7}{|l|}{ Faixa etária } \\
\hline $60-69$ & 1 & --- & & 1 & --- & \\
\hline 70-79 & 0,68 & $0,35-1,32$ & 0,255 & 0,41 & $0,20-0,87$ & 0,020 \\
\hline$\geq 80$ & 0,45 & $0,19-1,07$ & 0,071 & 0,17 & $0,05-0,55$ & 0,003 \\
\hline \multicolumn{7}{|l|}{ Sedentarismo } \\
\hline $\operatorname{Sim}$ & 1 & --- & & 1 & --- & \\
\hline Não & 1,09 & $0,58-2,04$ & 0,782 & 1,99 & $0,96-4,12$ & 0,064 \\
\hline \multicolumn{7}{|l|}{ Hiperglicemia } \\
\hline Sim & 1 & --- & & 1 & --- & \\
\hline Não & 1,18 & $0,58-2,42$ & 0,642 & 1,82 & $0,85-3,92$ & 0,126 \\
\hline \multicolumn{7}{|l|}{ LDL alto } \\
\hline Sim & 1 & --- & & 1 & --- & \\
\hline Não & 1,49 & $0,66-3,37$ & 0,339 & 2,20 & $0,94-5,19$ & 0,071 \\
\hline \multicolumn{7}{|c|}{ Hipertrigliceridemia } \\
\hline $\operatorname{Sim}$ & 1 & --- & & 1 & --- & \\
\hline Não & 1,33 & $0,73-2,43$ & 0,345 & 2,25 & $1,15-4,44$ & 0,019 \\
\hline
\end{tabular}

*Valores de p baseados em regressão logística múltipla; OR: odds ratio; IC: intervalo de confiança.

\section{DISCUSSÃO}

Dos idosos entrevistados neste estudo, $72 \%$ estavam acima do peso e a prevalência de obesidade foi maior nas mulheres $(35 \%)$ do que nos homens $(21 \%)$. Dalacorte et al. ${ }^{3}$ obtiveram resultados semelhantes quando avaliaram a relação entre síndrome metabólica e atividade física em idosos de um município da região metropolitana de Porto Alegre. A prevalência de obesidade foi de $36 \%$ em mulheres e $26 \%$ em homens. Estudo realizado na região metropolitana de Curitiba mostrou que $57 \%$ dos idosos estavam acima do peso, sendo a maior frequência $(61 \%)$ encontrada nas mulheres. ${ }^{14}$ Outro estudo com idosos, realizado no Estado do Paraná (Londrina), mostrou uma prevalência de obesidade de $19 \%$, sendo $24 \%$ nas mulheres e $9 \%$ nos homens. ${ }^{6}$ 
Nota-se que, nos estudos populacionais, a prevalência de obesidade é maior entre as mulheres. Vários fatores podem explicar esses achados: as mulheres acumulam mais gordura visceral e subcutânea do que os homens; há diferenças no padrão alimentar entre os sexos; as mulheres apresentam maior expectativa de vida; a menopausa é acompanhada por aumento de peso e adiposidade. ${ }^{15}$ Os resultados deste estudo corroboram outros estudos realizados com idosos, mostrando que o excesso de peso, e não a desnutrição, constitui o principal problema nutricional da população idosa brasileira. ${ }^{16,17}$

$\mathrm{Na}$ população estudada, o número de indivíduos obesos foi menor nas faixas etárias mais elevadas (>80 anos), sugerindo que a obesidade é um fator limitante para a longevidade. Em concordância com o estudo de revisão realizado por Villareal et al., ${ }^{18}$ indivíduos eutróficos apresentam maior expectativa de vida em relação àqueles com sobrepeso ou obesidade. Em uma amostra representativa de adultos de um município do interior do Estado do Rio Grande do Sul, houve aumento da prevalência de obesidade conforme o avanço da idade a partir dos 30 até os 70 anos de idade. ${ }^{4}$

De modo geral, poucos indivíduos obesos ultrapassam os 80 anos de idade. Cabrera \& Jacob Filho ${ }^{6}$ sugerem que a diminuição da obesidade acima dos 80 anos pode ocorrer devido à interferência da obesidade e das patologias a ela associadas, que são fatores que contribuem para maior mortalidade de idosos obesos antes dos 80 anos. Os autores também mencionam que o próprio processo de senescência poderia contribuir para explicar a menor prevalência de obesos com mais de 80 anos. Alguns autores sugerem que o idoso com idade mais avançada possui menor chance de apresentar sobrepeso, possivelmente devido às alterações na composição corporal. ${ }^{16}$ Segundo a OMS, a manutenção do estado nutricional adequado não significa necessariamente maior sobrevida, mas pode interferir positivamente, permitindo ao idoso alcançar seu ciclo máximo de vida. ${ }^{19}$
Exames bioquímicos como a glicemia e o perfil lipídico podem estar alterados no idoso, ao passo que modificações na qualidade nutricional da dieta podem reverter esse quadro. ${ }^{20} \mathrm{Na}$ população estudada, a hipertrigliceridemia foi mais frequente nas mulheres obesas. Em relação aos triglicerídeos séricos, não houve diferença estatisticamente significativa entre homens obesos e os demais grupos, possivelmente devido ao tamanho da amostra. Já a glicemia aumentou somente nas mulheres, provavelmente devido à obesidade, cuja prevalência foi maior no sexo feminino. Em 2006, um grande estudo envolvendo mais de 49 mil indivíduos de todas as capitais brasileiras mostrou que a prevalência de diabetes e dislipidemia é duas vezes maior em indivíduos com sobrepeso e aproximadamente três vezes maior nos obesos. ${ }^{21}$

Considerando o hábito alimentar da população da Região Sul do Brasil, este estudo mostra que há maior consumo de proteína pelos homens obesos. Conforme a tradição alimentar local do Rio Grande do Sul, a principal fonte de proteína na dieta é proveniente de carnes em geral (gado, frango, porco, ovelha, vísceras, linguiça, toucinho, etc.), cujo consumo é predominante em homens. Um estudo de coorte realizado nos Estados Unidos durante 20 anos concluiu que o consumo de carnes e proteína de origem animal está associado ao aumento da mortalidade por diversas causas, entre elas, doença coronariana, diabetes e câncer. ${ }^{22}$ Outro estudo de revisão, baseado nos resultados de seis estudos de coorte, concluiu que um baixo consumo de carne está associado ao aumento da longevidade. ${ }^{23}$ Pesquisa realizada com idosos centenários revelou que a maioria dos indivíduos seguia uma dieta equilibrada, contendo todos os grupos de alimentos, ou seja, frutas, vegetais, laticínios, grãos, cereais, óleos vegetais e carnes. ${ }^{13}$

Sabe-se que os produtos de origem animal são também as principais fontes de gordura saturada, composta principalmente de ácidos graxos de cadeia longa, como ácido palmítico, mirístico e esteárico, capazes de aumentar os 
níveis séricos de LDL e apo-lipoproteína B, além de diminuir a razão HDL/LDL. ${ }^{24}$ Assim, a dieta para o tratamento da hipercolesterolemia deve ser pobre em ácidos palmítico e mirístico, consumidos em grande quantidade na Região Sul do Brasil, onde o consumo excessivo de carne (churrasco) é responsável pela alta prevalência de obesidade, dislipidemia e doenças cardiovasculares nessa população. ${ }^{25}$

Neste estudo, mais da metade dos idosos obesos do sexo masculino apresentava HDL reduzido e hipertrigliceridemia. Em relação às mulheres, em todas as categorias avaliadas, mais da metade possuía hipercolesterolemia. Ao analisar somente as obesas, mais de 60\% delas apresentavam hipercolesterolemia, HDL reduzido e hipertrigliceridemia. Esses dados indicam a manutenção de uma dieta nutricionalmente desequilibrada, concordando com dados obtidos por Temme et al. ${ }^{26}$

Além dos fatores dietéticos, o aumento dos níveis de colesterol pode ser explicado pelas complicações metabólicas, nas quais ocorre desregulação do processo de lipólise, resultando em maior liberação de ácidos graxos e glicerol. Os ácidos graxos livres fornecem a maior parte do combustível lipídico circulante e seu excesso constitui a hiperlipidemia. $\mathrm{O}$ tecido adiposo é o maior reservatório de colesterol do organismo e essa quantidade aumenta com a idade e com o aumento do peso corporal. Dislipidemia e obesidade são muito frequentes, principalmente nos idosos. Esses dados são preocupantes, pois ambos são potenciais fatores de risco para várias doenças, com destaque para a doença aterosclerótica, motivo pelo qual necessitam de maior atenção, tanto por parte dos pacientes quanto dos sistemas de saúde. ${ }^{11}$

A obesidade é causa de diversas complicações que podem acometer diferentes sistemas, estando associada a problemas metabólicos, sanguíneos, urinários, respiratórios e ósseos. ${ }^{27} \mathrm{~A}$ fim de evitar o declínio funcional progressivo no idoso obeso, intervenções no estilo de vida devem ser propostas, tais como a redução da ingestão calórica diária combinada a um programa de exercícios físicos, preferencialmente aeróbico e de resistência. ${ }^{18}$

Na população estudada, mais da metade das mulheres idosas, nas três categorias avaliadas (normais, sobrepeso e obesas), não praticava atividade física. $\mathrm{O}$ mesmo não ocorreu no sexo masculino, já que a frequência de idosos sedentários foi maior entre os obesos. Outros estudos brasileiros mostram maior prevalência de obesidade entre os idosos sedentários, independentemente do sexo. ${ }^{3,6,17}$ Os resultados deste estudo sugerem que a alta prevalência de obesidade nos idosos de Porto Alegre se deve ao sedentarismo e ao elevado consumo de alimentos ricos em açúcar e gordura. No idoso, o exercício físico proporciona diminuição da sarcopenia, reduzindo também perda da densidade mineral óssea, o que, em conjunto, reduz a fragilidade e confere maior qualidade de vida ao idoso. ${ }^{28}$

Algumas limitações do estudo devem ser pontuadas, especialmente em relação ao método utilizado para avaliar a ingestão alimentar (inquérito recordatório de 24 horas). Não se pode assegurar que o consumo alimentar de um único dia reflete com precisão o hábito alimentar do indivíduo, motivo pelo qual associou-se a história dietética. Para maior acurácia na verificação do consumo de nutrientes, mais dias de inquéritos alimentares são necessários. Viés de subnotificação é frequentemente encontrado em estudos populacionais sobre consumo de alimentos..$^{29,30}$ A subnotificação de dados nos inquéritos alimentares ocorre sobretudo em indivíduos com sobrepeso e obesos, para os registros de lanches e sobremesas, alimentos de alta densidade calórica, frequência de consumo e tamanho das porções, podendo alcançar entre 30 e $50 \%$ de subnotificações. ${ }^{31}$

\section{CONCLUSÃO}

A manutenção do estado nutricional adequado no idoso é tarefa árdua frente ao hábito alimentar, estilo de vida, doenças crônicas, uso 
de medicamentos e modificações fisiológicas inerentes à idade. As mulheres apresentaram os maiores índices de sobrepeso e dislipidemia, ao passo que os homens foram os maiores consumidores de proteína na dieta. Além disso, mulheres e homens obesos foram os que menos praticam atividade física.

Esses achados contribuem para a alta prevalência de obesidade no Rio Grande do Sul, tornando urgente a atenção à obesidade e às consequências na saúde dos idosos, principalmente por programas vinculados

\section{REFERÊNCIAS}

1. Chaimowicz F. A saúde dos idosos brasileiros às vésperas do século XXI: problemas, projeções e alternativas. Rev Saude Pública 1997;31(2):184-200.

2. Paiva PTA, Wajnman S. Das causas às consequências econômicas da transição demográfica no Brasil. Rev Bras Estud Popul 2005;22(2):303-22.

3. Dalacorte RR, Reichert CL, Vieira JL. Metabolic syndrome and physical activity in southern Brazilian community-dwelling elders: a population-based, cross-sectional study. BMC Public Health 2009;9:25.

4. Sarturi JB, Neves J, Peres KG. Obesidade em adultos: estudo de base populacional num município de pequeno porte no sul do Brasil em 2005. Ciênc Saúde Coletiva 2010;15(1):105-13.

5. Obesity: preventing and managing the global epidemic. Report of a WHO consultation. World Health Organ Tech Rep Ser 2000;894:i-xii.

6. Cabrera MAS, Jacob Filho W. Obesidade em idosos: prevalência, distribuição e associação com hábitos e co-morbidades. Arq Bras Endocrinol Metab 2001;45(5):494-501.

7. Stevens J. Impact of age on associations between weight and mortality. Nutr Rev 2000;58(5):129-37.

8. Santos DM, Sichieri R. Índice de massa corporal e indicadores antropométricos de adiposidade em idosos. Rev Saúde Pública 2005;39(2):163-8.

9. World Health Organization. Estimated Obesity Prevalence: aged 15+, 2010 [acesso em 1 dez 2010]. Disponível em: https://apps.who.int/infobase/ Comparisons.aspx ao sistema público de saúde. Com orientação especializada, a obesidade poderá ser prevenida e tratada com efetividade.

\section{AGRADECIMENTOS}

Prefeitura Municipal de Porto Alegre, RS; ao Hospital São Lucas da PUCRS; ao Instituto de Geriatria e Gerontologia; à Coordenação de Aperfeiçoamento de Pessoal de Nível Superior (CAPES). Ao colega Dr. Eduardo Lopes Nogueira, pelas contribuições e sugestões estatísticas prestadas neste trabalho.

10. Instituto Brasileiro de Geografia e Estatística.

Prevalência de déficit de peso, excesso de peso e obesidade na população com 20 ou mais anos de idade, por sexo, segundo Unidades da Federação, áreas urbanas dos Municípios das Capitais e Regiões Metropolitanas - período 2002-2003 [acesso em 1 dez 2010]. Disponível em: http://www.ibge.gov. br/home/estatistica/populacao/condicaodevida/ pof/2002analise/tab01e.pdf.

11. Nagatsuyu DT, Moriguti EKU, Pfrimer K, Formighieri PF, Lima NKC, Ferriolli E, et al. O impacto da obesidade abdominal sobre os níveis plasmáticos de lípides nos idosos. Medicina 2009;42(2):157-63.

12. Huang KC, Lee MS, Lee SD, Chang YH, Lin YC, Tu $\mathrm{SH}$, et al. Obesity in the elderly and its relationship with cardiovascular risk factors in Taiwan. Obes Res 2005;13(1):170-8.

13. Busnello FM. Padrão alimentar e a longevidade em centenários de Porto Alegre. [tese de mestrado]. Porto Alegre: Instituto de Geriatria e Gerontologia da Pontifífia Universidade Católica; 2005.

14. Bassler TC, Lei DLM. Diagnóstico e monitoramento da situação nutricional da população idosa em município da região metropolitana de Curitiba (PR). Rev Nutr 2008;21(3):311-21.

15. Silveira EA, Kac G, Barbosa LS. Prevalência e fatores associados à obesidade em idosos residentes em Pelotas, Rio Grande do Sul, Brasil: classificação da obesidade segundo dois pontos de corte do índice de massa corporal. Cad Saúde Pública 2009;25(7):1569-77. 
16. Campos MAG, Pedroso ERP, Lamounier JA, Colosimo EA, Abrantes MM. Estado nutricional e fatores associados em idosos. Rev Assoc Med Bras 2006;52(4):214-21.

17. Barreto SM, Passos VMA, Lima-Costa MFF. Obesidade e baixo peso entre idosos brasileiros. Projeto Bambuí. Cad Saúde Pública 2003;19(2):605-12.

18. Villareal DT, Apovian CM, Kushner RF, Klein S. Obesity in older adults: technical review and position statement of the American Society for Nutrition and NAASO, The Obesity Society. Am J Clin Nutr 2005;82(5):923-34.

19. Physical status: the use and interpretation of anthropometry. Report of a WHO Expert Committee. World Health Organ Tech Rep Ser 1995;854:1-452.

20. Buyken AE, Flood V, Rochtchina E, Nestel P, BrandMiller J, Mitchell P. Modifications in dietary fat quality are associated with changes in serum lipids of older adults independently of lipid medication. J Nutr 2010;140(1):88-94.

21. Gigante DP, Moura EC, Sardinha LMV. Prevalence of overweight and obesity and associated factors, Brazil, 2006. Rev Saude Publica 2009:43(Supl. 2):83-9.

22. Snowdon DA. Animal product consumption and mortality because of all causes combined, coronary heart disease, stroke, diabetes, and cancer in Seventhday Adventists. Am J Clin Nutr 1988;48(3):739-48.

23. Singh PN, Sabaté J, Fraser GE. Does low meat consumption increase life expectancy in humans? Am J Clin Nutr 2003;78(3):526S-32.

24. Zock PL, de Vries JH, Katan MB. Impact of myristic acid versus palmitic acid on serum lipid and lipoprotein levels in healthy women and men. Arterioscler Thromb 1994;14(4):567-75.

25. Monteiro CA, Mondini L, Costa RBL. Mudanças na composição e adequação nutricional da dieta familiar nas áreas metropolitanas do Brasil (1988-1996). Rev Saúde Pública 2000;34(3):251-8.

26. Temme E, Huybrechts I, Vandevijvere S, De Henauw S, Leveque A, Kornitzer M, et al. Energy and macronutrient intakes in Belgium: results from the first National Food Consumption Survey. Br J Nutr 2010;103(12):1823-9.

27. Jensen GL. Obesity and functional decline: epidemiology and geriatric consequences. Clin Geriatr Med 2005;21(4):677-87.

28. Binder EF, Schechtman KB, Ehsani AA, Steger-May K, Brown M, Sinacore DR, et al. Effects of exercise training on frailty in community-dwelling older adults: results of a randomized, controlled trial. J Am Geriatr Soc 2002;50(12):1921-8.

29. Huang TT, Roberts SB, Howarth NC, McCrory MA. Effect of screening out implausible energy intake reports on relationships between diet and BMI. Obes Res 2005;13(7):1205-17.

30. Poppitt SD, Swann D, Black AE, Prentice AM. Assessment of selective under-reporting of food intake by both obese and non-obese women in a metabolic facility. Int J Obes Relat Metab Disord 1998;22(4):303-11.

31. Lutomski JE, van den Broeck J, Harrington J, Shiely F, Perry IJ. Sociodemographic, lifestyle, mental health and dietary factors associated with direction of misreporting of energy intake. Public Health Nutr 2011;14(3):532-41. 